\title{
Island-assisted interface alloying and magnetic polarization at submonolayer $\mathrm{V} / \mathrm{Cr}(001)$ interfaces
}

\author{
C. Clavero, ${ }^{1, *}$ M. Bode,${ }^{2}$ G. Bihlmayer ${ }^{3}$ S. Blügel,${ }^{3}$ and R. A. Lukaszew ${ }^{1,4}$ \\ ${ }^{1}$ Department of Applied Science, The College of William \& Mary, Williamsburg, Virginia 23187, USA \\ ${ }^{2}$ Center for Nanoscale Materials, Argonne National Laboratory, Argonne, Illinois 60439, USA \\ ${ }^{3}$ Institut für Festkörperforschung and Institute for Advanced Simulation, Forschungszentrum Jülich, D-52425 Jülich, Germany \\ ${ }^{4}$ Department of Physics, The College of William \& Mary, Williamsburg, Virginia 23187, USA \\ (Received 28 June 2010; revised manuscript received 5 August 2010; published 27 August 2010)
}

\begin{abstract}
Island-assisted interface alloying was observed during submonolayer deposition on $\mathrm{Cr}(001)$ substrates at 525 K. Scanning tunneling spectroscopy suggests atomic interchange at the center of the islands during the early stages of growth, giving rise to a $\mathrm{Cr}$ core in the center of the island and a gradually increasing $\mathrm{V}$ concentration toward the island rim. The existence of a $\mathrm{VCr}$ alloy with equiatomic composition is concluded by comparing tunneling spectra measured at the island rim with density-functional theory calculations. Coalescence of the initial islands gives rise to inhomogeneous alloying at monolayer coverage. Antiferromagnetic coupling between the islands and the $\mathrm{Cr}(001)$ substrate is found for coverages up to 0.50 atomic layers. At higher coverages, no magnetic contrast was observed.
\end{abstract}

DOI: 10.1103/PhysRevB.82.085445

PACS number(s): 75.70.-i, 68.37.Ef, 68.55.-a

\section{INTRODUCTION}

While interfaces between ferromagnetic and nonmagnetic metals have attracted considerable attention in the field of magnetism, the growth and magnetic polarization of nonmagnetic metals on antiferromagnets has remained largely unexplored. This is particularly surprising since proximity effects such as magnetic polarization of nonmagnetic elements, ${ }^{1}$ interfacial alloying, ${ }^{2}$ and interdiffusion ${ }^{3}$ do not only play a significant role in the design of artificial superlattices with potentially novel properties but are also of fundamental importance in practical applications such as the use of the exchange bias effect in perpendicular magnetic recording media, ${ }^{4}$ giant magnetoresistance sensors,${ }^{5}$ and random access memories. ${ }^{6}$

Of special interest has been the $\mathrm{V} / \mathrm{Cr}(001)$ interface, which is still controversially discussed and has attracted considerable attention in recent years. ${ }^{7-13}$ Both $\mathrm{V}$ and $\mathrm{Cr}$ are bcc $3 d$ transition metals with approximately half-band filling. $\mathrm{Cr}$ exhibits antiferromagnetism along the $\langle 001\rangle$ direction such that the (001) planes couple antiferromagnetically from layer to layer. It has been theoretically predicted that a single $\mathrm{V}$ layer on $\mathrm{Cr}$ would acquire an induced $\mathrm{V}$ moment of $2.1 \mu_{\mathrm{B}}$ and should couple antiferromagnetically to $\mathrm{Cr}$ while at the same time drastically reducing the $\mathrm{Cr}$ magnetic moment at the interface. ${ }^{7,8,12,13}$ Indeed, Mibu et al. ${ }^{10,14}$ have provided experimental support for the reduction in the $\mathrm{Cr}$ magnetic moment at the interface using Mössbauer spectroscopy. Furthermore, recent neutron-scattering experiments have shown that proximity effects at $\mathrm{Cr} / \mathrm{V}$ interfaces lead to the appearance of a $50 \AA$ wide magnetically dead $\mathrm{Cr}$ layer near the $\mathrm{Cr} / \mathrm{V}$ interface, the suppression of the spin-flip transition, and the stabilization of a single-domain spin-density wave (SDW) in Cr with out-of-plane spins and out-of-plane propagation wave vector. ${ }^{9,15}$ While the reduction in the Cr magnetic moment has been demonstrated experimentally, the magnetic state of $\mathrm{V}$ in contact with antiferromagnetic $\mathrm{Cr}(001)$ remains unexplored and unclear. The observation of the $\mathrm{V}$ magnetic state at the interface with $\mathrm{Cr}(001)$ is challenging since the magnetic moments of adjacent antiparallel layers compensate and cannot be detected with spatially averaging methods.

Here we present our experimental and theoretical studies to unravel the structural and magnetic properties of $\mathrm{V}$ islands deposited onto $\mathrm{Cr}(001)$ substrates. By combining scanning tunneling microscopy (STM) and spectroscopy (STS) with density-functional theory (DFT) calculations we show that V growth on $\mathrm{Cr}(001)$ at $525 \mathrm{~K}$ leads to island-assisted alloying between the substrate and the islands in contrast to the expected formation of a chemically abrupt interface. ${ }^{9,10,14,15} \mathrm{~A}$ radial concentration gradient can be observed during the early stages of growth with a high $\mathrm{Cr}$ concentration in the island center and a gradually increasing $\mathrm{V}$ concentration toward the island rim. Upon coalescence this gradient across the islands leads to a monolayer with inhomogeneous composition. We show that the islands are antiferromagnetically coupled to the $\mathrm{Cr}(001)$ substrate at low coverage using spinpolarized STM (SP-STM). We find no magnetic contrast for coverages exceeding 0.5 atomic layers (AL) of V, possibly due to the quenching of the $\mathrm{Cr}$ spin-density wave in the surface near region.

\section{EXPERIMENTAL}

The experiments were carried out in an ultrahigh-vacuum system consisting of a preparation chamber for tip and sample treatment and an analysis chamber for surface characterization. A polycrystalline $\mathrm{W}$ tip was electrochemically etched ex situ and cleaned in vacuo by a high-temperature flash at $T=2200 \mathrm{~K}$. Subsequently, e-beam evaporation of a thin Fe film from a carefully degassed Fe rod was performed onto the tip apex. Fe-coated tips are preferentially magnetized perpendicular to the tip axis, leading to sensitivity to the in-plane component of the samples surface magnetization. ${ }^{16}$ Prior to the growth, the $\mathrm{Cr}(001)$ single crystal was cleaned overnight by $\mathrm{Ar}^{+}$-ion etching at elevated temperatures $(T \approx 1100 \mathrm{~K})$ and subsequently annealed for 20-30 min at $T=1150 \mathrm{~K}$. V was e-beam evaporated at an approximate rate of $0.3 \mathrm{AL} / \mathrm{min}$ on $\mathrm{Cr}(001)$. The substrate 


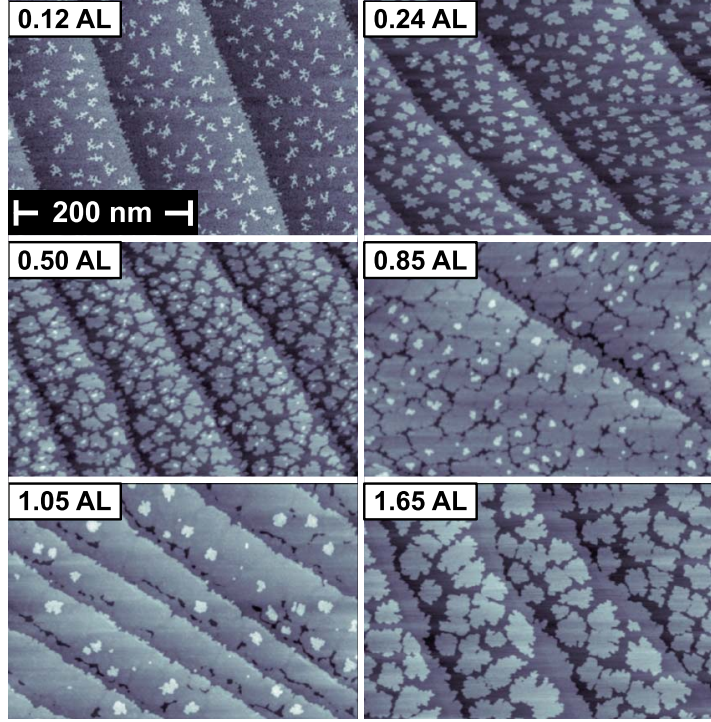

FIG. 1. (Color online) Topography STM maps of V islands deposited on $\mathrm{Cr}(001)$ substrates at $525 \mathrm{~K}$ with coverages from 0.12 to 1.65 AL. Layer-by-layer growth is observed.

deposition temperature during growth was $525 \mathrm{~K}$, which reportedly leads to abrupt interfaces. ${ }^{9,10,14,15}$ In this study, V coverages ranging from 0.12 to $1.65 \mathrm{AL}$ where explored. The SP-STM measurements were performed at $157 \mathrm{~K}$ using a STM system attached to the analysis chamber. The base pressure in the measurement system was in the low $10^{-11}$ Torr range. The STS measurements were performed by adding a modulation voltage $U_{\text {mod }}=20 \mathrm{mV}_{\text {rms }}$ to the applied sample bias $U$ and recording the $d I / d U$ signal using lock-in techniques.

\section{GROWTH MODE AND ISLAND-ASSISTED ALLOYING}

Figure 1 shows topographic STM images of islands after the deposition of various amounts of $\mathrm{V}$ on $\mathrm{Cr}(001)$. The coverages range from 0.12 to $1.65 \mathrm{AL}$. During the early stages of growth, nucleation of islands with monoatomic height is observed on the $\operatorname{Cr}(001)$ substrate and close to the terrace edges. After further deposition, the island size coarsens until coalescence at coverages around $0.65-0.85 \mathrm{AL}$. Although the growth of $\mathrm{V}$ proceeds almost perfectly in a layer-by-layer mode, the nucleation of small second-layer islands was observed at coverages as low as $0.5 \mathrm{AL}$. The nucleation center of these second-layer islands occurred predominantly over the center of the supporting underlying monolayer islands. However, coarsening of these second-layer islands was found to be very slow while the first layer was yet not complete.

STS has the ability to probe the local density of states (DOS) through $d I / d U$ conductivity curves and allows the detection of potential chemical inhomogeneities across topologically homogeneous surface areas. Figure 2(a) shows differential conductance curves measured on the substrate and at different positions on the island; the sample's average $\mathrm{V}$ coverage amounts to $0.09 \mathrm{AL}$ [see Fig. 2(b) for a topographic (a)
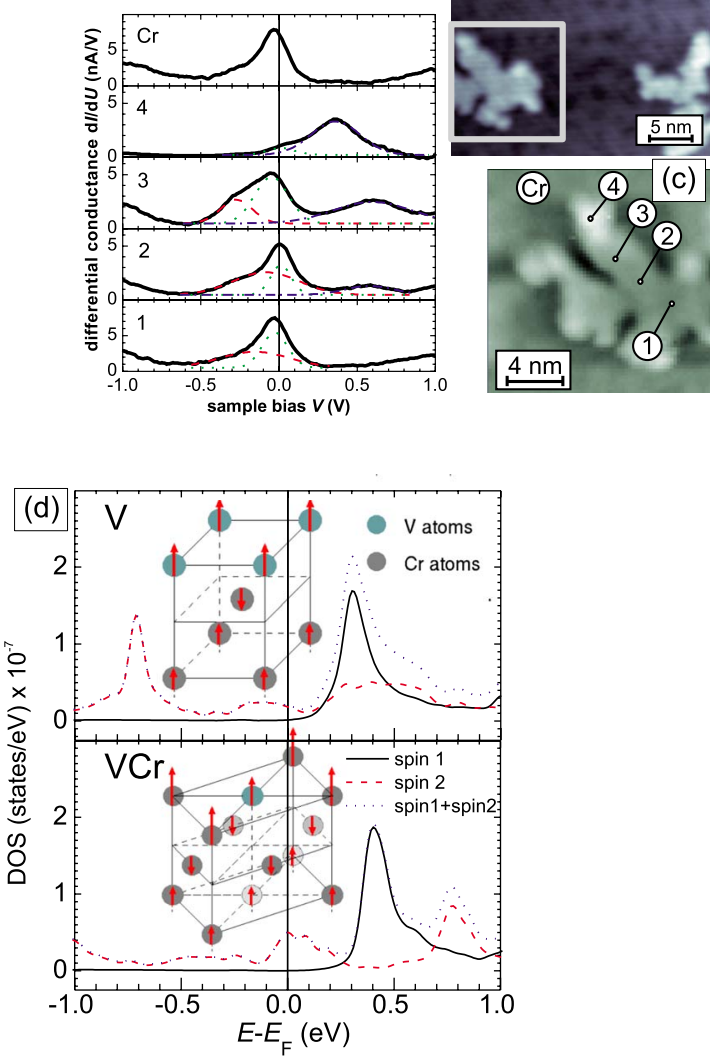

FIG. 2. (Color online) (a) Differential conductance curves measured on the substrate at different positions on one of the islands for 0.09 AL coverage [see (b) and (c) for a topographic images]. A Gaussian fitting of the $d I / d U$ curves shows the different contributions (dotted lines). (d) DOS simulations for a single $\mathrm{V}$ AL on $\mathrm{Cr}(001)$ and for a single $\mathrm{AL}$ of equiatomic $\mathrm{CrV}$ alloy on $\mathrm{Cr}(001)$.

image]. Within the smaller scan frame, shown in Fig. 2(c), a $d I / d U$ spectrum has been measured at every pixel $(0.25$ $\times 0.25 \mathrm{~nm}^{2}$ ). The positions where the spectra of Fig. 2(a) have been recorded are labeled consistently. The $\operatorname{Cr}(001)$ substrate exhibits a single peak centered at $U=-0.015 \mathrm{~V}$ which is well known for bcc(001) surfaces. However, the physical origin of this state is still controversial. ${ }^{17,18}$ Originally interpreted as a $d_{z^{2}}$-like surface state at energies close to the Fermi level,,$^{19}$ an alternative explanation is an orbital Kondo resonance. ${ }^{20}$ Although the exact nature of the electronic feature is unclear, it can serve as a characteristic marker for the presence of Cr. Interestingly, a single peak centered at $U=-0.015 \mathrm{~V}$ was also found in the spectrum taken on the center of the island (see spectrum 1). This result suggests that the island center consists of $\mathrm{Cr}$ rather than $\mathrm{V}$, possibly caused by interdiffusion. It is worth noticing that the vacuum DOS of a system consisting of a buried V layer in $\mathrm{Cr}$ was calculated and found to be very similar to the pure $\mathrm{Cr}(001)$. For spectra taken at an increasing distance from the island center, the Fermi-level peak intensity decreases while a second peak initially centered in $U=0.6 \mathrm{~V}$ appears and gradually shifts to lower bias voltages (spectra 2-4). This second peak is predominant at the rim of the islands and it is 
found at $U=0.4 \mathrm{~V}$. Previous reports have shown that the surface states features of $\mathrm{Cr}(001)$ (Ref. 21) and $\mathrm{V}(001)$ (Ref. 22) shift toward higher energies when impurities are incorporated into the lattice. In addition, a two-peaks feature found in the STS spectra of $\mathrm{Fe} / \mathrm{Cr}$ systems has also been associated to surface alloying.,23

At a first glance, our observations indicating interdiffusion between $\mathrm{V}$ and the $\mathrm{Cr}$ substrate are quite surprising since theoretical calculations find a segregation energy of $-180 \mathrm{meV} /$ surface atom. ${ }^{24}$ The negative energy suggests that a monolayer of $\mathrm{V}$ surface should be energetically stable on the $\mathrm{Cr}(001)$. However, published experimental and theoretical data have been found to be inconsistent for other material combinations too, indicating possible fundamental problems with computing segregation energies for $\mathrm{Cr}$ and similar $3 d$ metals. For example, for $\mathrm{Fe}$ on $\mathrm{Cr}$ an even larger negative segregation energy of $-590 \mathrm{meV} /$ surface atom has been calculated. ${ }^{24}$ In contrast, experimental data clearly show strong interdiffusion at similar deposition temperatures. ${ }^{3,23}$

In order to better understand the observed evolution in the spectra and the related composition of the islands, we have carried out vacuum DOS calculations using DFT within the generalized gradient approximation ${ }^{25}$ and the full-potential linearized augmented plane-wave method. ${ }^{26}$ Figure 2(d) upper panel shows the DOS for a $\mathrm{V}$ monolayer relaxed on a 21 layer film of $\mathrm{Cr}(001)$. Two characteristic peaks at -0.7 and $0.3 \mathrm{eV}$ are found. These theoretical data are in contrast with our observation since no peaks were found in the STS spectra at negative bias. This rules out the presence of pure $\mathrm{V}$ and points to alloying in the present case. Figure 2(d) lower panel shows the calculated DOS for a monolayer of equiatomic $c(2 \times 2) \mathrm{CrV}$ alloy on $\mathrm{Cr}(001)$, exhibiting a predominant peak at $U=0.4 \mathrm{~V}$, similar to the one found at the rim of the islands. This corroborates island-assisted interface alloying where the island $\mathrm{V}$ concentration is negligible at the core and gradually increases as the radial distance increases up to equiatomic $\mathrm{CrV}$ alloy composition at the rim. This mechanism is in contrast with other reported interfacial alloying observed at $\mathrm{Cr}-\mathrm{Fe}(001)$ interfaces as in the latter case the observed process was interpreted as a distribution of single atomic $\mathrm{Cr}$ impurities dispersed in the $\mathrm{Fe}$ substrate. ${ }^{2,3}$ Nevertheless, similar findings have been reported for the twodimensional adisland-assisted surface alloying of $\mathrm{Ni}$ on $\mathrm{Au} .{ }^{27}$

\section{MAGNETIC POLARIZATION: V/CR ANTIFERROMAGNETIC COUPLING}

In order to investigate the magnetic state of the islands, SP-STM measurements were carried out using Fe-coated W tips. Depending on the bias voltage selected during the measurements, differential conductivity $d I / d U$ mapping showed either magnetic or chemical contrast. For this experiment, magnetic contrast is obtained preferentially when small negative bias voltages are applied whereas chemical contrast is obtained for positive voltage, similar to previous reports using $\mathrm{Cr}(001)$ substrates. ${ }^{23,28}$ Figure 3 shows (a) the topography of a sample with $0.24 \mathrm{AL} \mathrm{V}$ coverage, and $d I / d U$ maps measured at (b) $U=+0.4 \mathrm{~V}$ and (c) $U=-0.4 \mathrm{~V}$, which
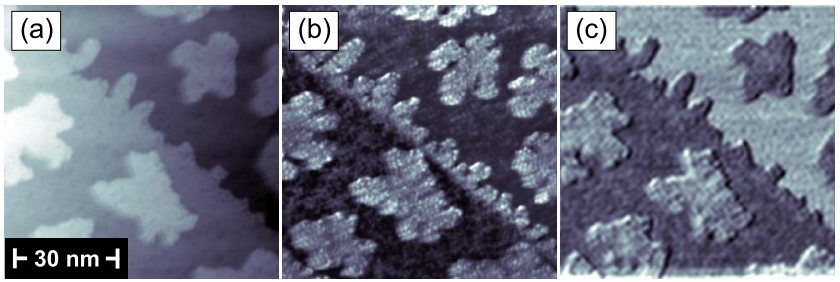

FIG. 3. (Color online) (a) Topography, (b) chemical contrast $d I / d U$ map measured at a bias voltage of $U=+0.4 \mathrm{~V}$, and (c) spinresolved $d I / d U$ map measured at $U=-0.4 \mathrm{~V}$ corresponding to the same area of a sample with 0.24 AL V coverage. Clearly, antiferromagnetic coupling is found between substrate and islands.

exhibit chemical contrast and magnetic contrasts, respectively. Due to their higher differential conductivity close to the $\mathrm{VCr}$ peak position of $+0.4 \mathrm{~V}$, the chemical contrast image shows a lighter contrast for the islands, illustrating their position. The magnetization direction should alternate between adjacent terraces due to the topological antiferromagnetic order of the $\mathrm{Cr}(001)$ terrace. $^{28}$ Indeed, alternating $d I / d U$ intensities are observed in the spin-resolved image [Fig. 3(c)] due to spin-polarized electron tunneling between the magnetic tip and the $\mathrm{Cr}$ substrate. Furthermore, a clear contrast between islands on different terraces can be observed, with dark islands being found on bright terraces and vice versa. This clearly demonstrates magnetic polarization of the islands which is antiparallel to the underlying $\mathrm{Cr}$ substrate. We note that even though the islands exhibit chemical inhomogeneity, their magnetic contrast is largely constant, demonstrating that the $\mathrm{Cr}$ and $\mathrm{V}$ atoms in the alloy are coupled antiferromagnetically with the substrate, in agreement with the fact that a ferromagnetic coupling of the alloy layer with the substrate could not be stabilized in the calculations.

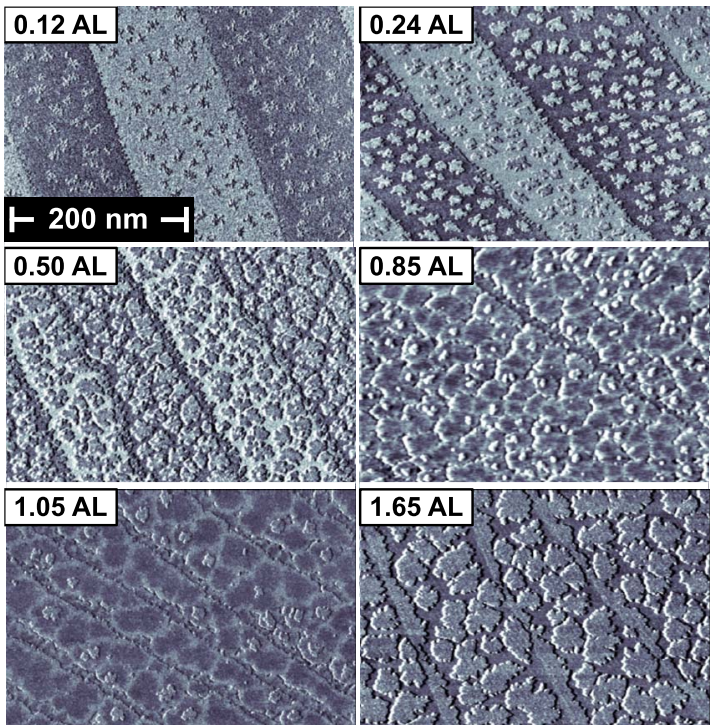

FIG. 4. (Color online) Spin resolved $d I / d U$ maps of the V films deposited on $\mathrm{Cr}(001)$ at $525 \mathrm{~K}$ with coverages from 0.12 to $1.65 \mathrm{AL}$ measure with a bias voltage of $-0.4 \mathrm{~V}$. Antiferromagnetic coupling between the Cr substrate and the islands grown on top is clearly observed for coverages up to $0.85 \mathrm{AL}$. 
Figure 4 shows spin-resolved $d I / d U$ maps measured simultaneously with the topographic data shown in Fig. 1. The bias voltage for all images was $U=-0.4 \mathrm{~V}$. Neighboring $\mathrm{Cr}$ terraces show an alternating intensity in the differential conductivity $d I / d U$ at low coverages between 0.12 and $0.50 \mathrm{AL}$, in agreement with the well-known topological antiferromagnetic order of $\mathrm{Cr}(001) .{ }^{28}$ At the same time, a clear contrast between islands on different terraces for coverages up to 0.50 $\mathrm{AL}$ can be recognized. The radial contrast due to the coexistence of $\mathrm{Cr}$ - and $\mathrm{CrV}$-dominated areas, best visible in the image showing 1.05 AL in Fig. 4, remains at higher coverages. Evidently, the inhomogeneous composition observed in separate islands [cf. Figs. 2(a)-2(c)] is maintained even after coalescence of the islands. Thus, we found evidence for a new alloying mechanism which originates from atomic interchange during the initial stages of island growth followed by island coalescence with no significant composition modification at higher coverages. For these higher coverages, however, the magnetic contrast on both the terraces and the islands starts to vanish. This is consistent with previous studies where the onset of a magnetically dead layer close to the $\mathrm{Cr} / \mathrm{V}$ interface was observed $\mathrm{d}^{9,10,14,15}$ in thick enough $\mathrm{V}$ films. This fact is attributed to the $\mathrm{Cr}-\mathrm{V}$ interface hybridization, which causes a short-range suppression of the SDW magnetism near the $\mathrm{Cr} / \mathrm{V}$ interfaces, leading to pinning of SDW nodes at the interfaces, as theoretically predicted. ${ }^{29}$

\section{CONCLUSIONS}

Our complete experimental and theoretical study using STS, SP-STM, and band theory applied to submonolayer V deposited on $\mathrm{Cr}(001)$ substrates at $525 \mathrm{~K}$ reveals an islandassisted alloying process during the early stages of growth. Initially, the islands exhibit a radial $\mathrm{Cr}-\mathrm{V}$ concentration gradient. Subsequent growth leads to coalescence giving rise to islands with heterogeneous alloy composition. At low coverage, the alloy islands are antiferromagnetically coupled with the $\mathrm{Cr}(001)$ substrate. The reported island-assisted growth mode opens a frontier in the fundamental understanding of the alloying process in metallic interfaces in general, and particularly in the growth mode and magnetic state of nonmagnetic metals on antiferromagnetic interfaces during the early stages of growth.

\section{ACKNOWLEDGMENTS}

This work was partially supported by NSF-DMR under Grant No. 0605661, and the Research Corporation. Use of the Center for Nanoscale Materials at Argonne National Laboratory was supported by the U. S. Department of Energy, Office of Science, Office of Basic Energy Sciences, under Contract No. DE-AC02-06CH11357.

*cclavero@wm.edu

${ }^{1}$ Y. Suzuki, T. Katayama, P. Bruno, S. Yuasa, and E. Tamura, Phys. Rev. Lett. 80, 5200 (1998).

${ }^{2}$ A. Davies, J. A. Stroscio, D. T. Pierce, and R. J. Celotta, Phys. Rev. Lett. 76, 4175 (1996).

${ }^{3}$ Y. J. Choi, I. C. Jeong, J. Y. Park, S. J. Kahng, J. Lee, and Y. Kuk, Phys. Rev. B 59, 10918 (1999).

${ }^{4}$ D. Weller and A. Moser, IEEE Trans. Magn. 35, 4423 (1999).

${ }^{5}$ A. Deac, K. J. Lee, Y. Liu, O. Redon, M. Li, P. Wang, J. P. Nozires, and B. Dieny, J. Magn. Magn. Mater. 290-291, 42 (2005).

${ }^{6}$ S. Tehrani, J. M. Slaughter, E. Chen, M. Durlam, J. Shi, and M. DeHerren, IEEE Trans. Magn. 35, 2814 (1999).

${ }^{7}$ G. Bihlmayer, T. Asada, and S. Blügel, Phys. Rev. B 62, R11937 (2000).

${ }^{8}$ B. A. Hamad and J. M. Khalifeh, Surf. Sci. 492, 161 (2001).

${ }^{9}$ E. Kravtsov et al., Phys. Rev. B 76, 024421 (2007).

${ }^{10}$ M. Almokhtar, K. Mibu, A. Nakanishi, T. Kobayashi, and T. Shinjo, J. Phys.: Condens. Matter 12, 9247 (2000).

${ }^{11}$ A. Liebig, G. Andersson, J. Birch, and B. Hjrvarsson, Thin Solid Films 516, 8468 (2008).

${ }^{12}$ A. Boussendel and A. Haroun, Thin Solid Films 325, 201 (1998).

${ }^{13}$ A. Kellou, N. E. Fenineche, A. Tadjer, and H. Aourag, Mater. Chem. Phys. 80, 215 (2003).

${ }^{14}$ K. Mibu, M. Almokhtar, A. Nakanishi, T. Kobayashi, and T. Shinjo, J. Magn. Magn. Mater. 226-230, 1785 (2001).

${ }^{15}$ E. Kravtsov, A. Nefedov, F. Radu, A. Remhof, H. Zabel, R. Brucas, B. Hjorvarsson, A. Hoser, and S. B. Wilkins, Phys. Rev. B 70, 054425 (2004).

${ }^{16}$ M. Bode, Rep. Prog. Phys. 66, 523 (2003).

${ }^{17}$ T. Hänke, M. Bode, S. Krause, L. Berbil-Bautista, and R. Wiesendanger, Phys. Rev. B 72, 085453 (2005).

${ }^{18}$ M. Budke, T. Allmers, M. Donath, and M. Bode, Phys. Rev. B 77, 233409 (2008).

${ }^{19}$ J. A. Stroscio, D. T. Pierce, A. Davies, R. J. Celotta, and M. Weinert, Phys. Rev. Lett. 75, 2960 (1995).

${ }^{20}$ O. Y. Kolesnychenko, R. de Kort, M. I. Katsnelson, A. I. Lichtenstein, and H. van Kempen, Nature (London) 415, 507 (2002).

${ }^{21}$ M. Schmid, M. Pinczolits, W. Hebenstreit, and P. Varga, Surf. Sci. 377-379, 1023 (1997)

${ }^{22}$ M. M. J. Bischoff, C. Konvicka, A. J. Quinn, M. Schmid, J. Redinger, R. Podloucky, P. Varga, and H. van Kempen, Phys. Rev. Lett. 86, 2396 (2001)

${ }^{23}$ R. Ravlić, M. Bode, and R. Wiesendanger, J. Phys.: Condens. Matter 15, S2513 (2003).

${ }^{24}$ A. Christensen, A. V. Ruban, P. Stoltze, K. W. Jacobsen, H. L. Skriver, J. K. Norskov, and F. Besenbacher, Phys. Rev. B 56, 5822 (1997).

${ }^{25}$ J. P. Perdew, K. Burke, and M. Ernzerhof, Phys. Rev. Lett. 77, 3865 (1996).

${ }^{26} \mathrm{http}: / /$ www.flapw.de

${ }^{27}$ M. B. Hugenschmidt, A. Hitzke, and R. J. Behm, Phys. Rev. Lett. 76, 2535 (1996)

${ }^{28}$ M. Kleiber, M. Bode, R. Ravlic, N. Tezuka, and R. Wiesendanger, J. Magn. Magn. Mater. 240, 64 (2002).

${ }^{29}$ S. Demuynck, J. Meersschaut, J. Dekoster, B. Swinnen, R. Moons, A. Vantomme, S. Cottenier, and M. Rots, Phys. Rev. Lett. 81, 2562 (1998). 